\title{
Effect of Affective Computing Model on Distance Physical
}

\author{
Education
}

\author{
Zhen You and Shangling Song \\ Jining Polytechnic College, Shandong, China
}

\begin{abstract}
: to solve the problem of emotion absence on modern distance educatio $\mathrm{n}$, an affective computing model based on expression recognition was proposed, whose theoretic foundation was affective computing and whose kernel technol ogy was to estimate and comprehend learner's emotion states by catching and $r$ ecognizing their motion. corresponding affective encouragement and affective co mpensability strategies was proposed based on their given emotion states. The distance physical education based on affective computing model not only prom otes HCI and sloves the problem of the emotion absence, it can also realize th e Harmonious HCI in modern distance education.
\end{abstract}

Keywords:Modern Distance Education; Affective Computing;Motion recognition

\section{INTRODUCTION}

Modem distance education is important direction of the international education development since 1980s. It is a primary approach to realize information education and education internationalization, though modern distance education arouses a profound revolution in educational model and educational conception, moreover, the technologies and instruments on which it relied are advanced, yet it exists some shortcoming in several aspects,such as: notion orientation, network system and alternation etc, especially in alternation aspect,the space-time separate of modern distance education has learners could not satisfy their need in emotion communion, so emotion is absent. These absence badly influences learner's cognitive learning and mental health. Therefore, compensating emotion has most important significance for ensuring instructional quality and improving instructional effect of modern distance education. Affective computing is a hotspot problem in information science, cognition science and psychology studies, it bases on human being's emotion and affection, studies emotion mechanism, affective signal capturing, affective mode recognizing, emotion modeling and comprehending, emotion synthesizing and expressing, emotion transferring and alternating, and so on. Its final aim is endowing computer with the affective ability similarly with human beings.

Emotional model

Emotion model will be described by Figure 1, the producing mechanism of human behavior 
mainly depends on the external stimuli and the existing real-time emotion, so considering this issue on change mechanism change of the emotion, consideration must be given to external stimuli and with feelingssignal at the time. Emotional learning process mainly completed by the amygdala.

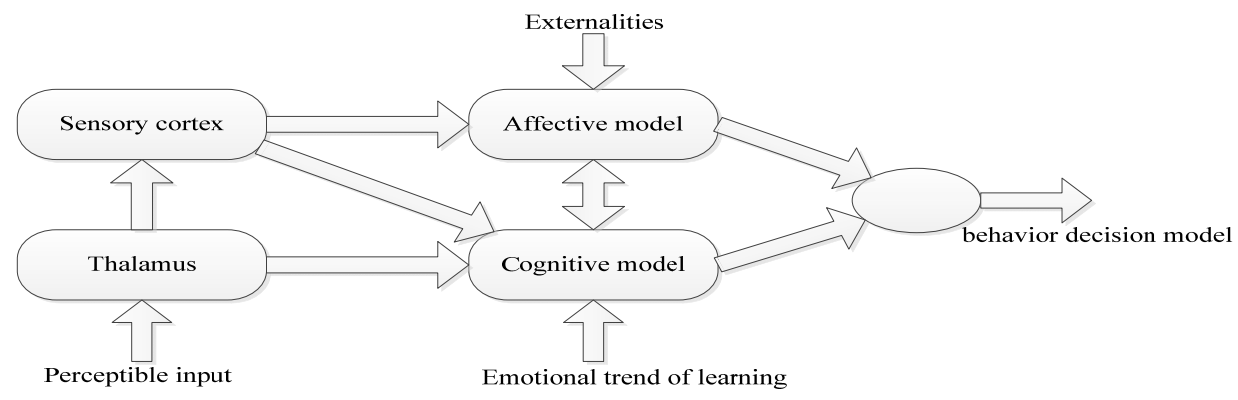

Fig 1 Emotion model

Emotional design is a kind of emotional designing concept that is developed in USA, this concept thought that the true value of products is to meet the user's emotional needs, the purpose of which is to make emotion into products through a variety of shapes, colors, mechanism and other designing elements, when consumers use the products, people can get the spirit of joy and feel satisfied and pleased, stimulate people's imagination and even produce resonance. Professor Donald Norman thought, design can be divided into three levels: automatic pre-set layer, which can also be called as Viscerallevel; the level contains the control behavior of common brain activity, which also can be called as Behaviorallevel; the level of brain's thinking, which also can be called as Refleetivelevel, shown in Fig.2: 


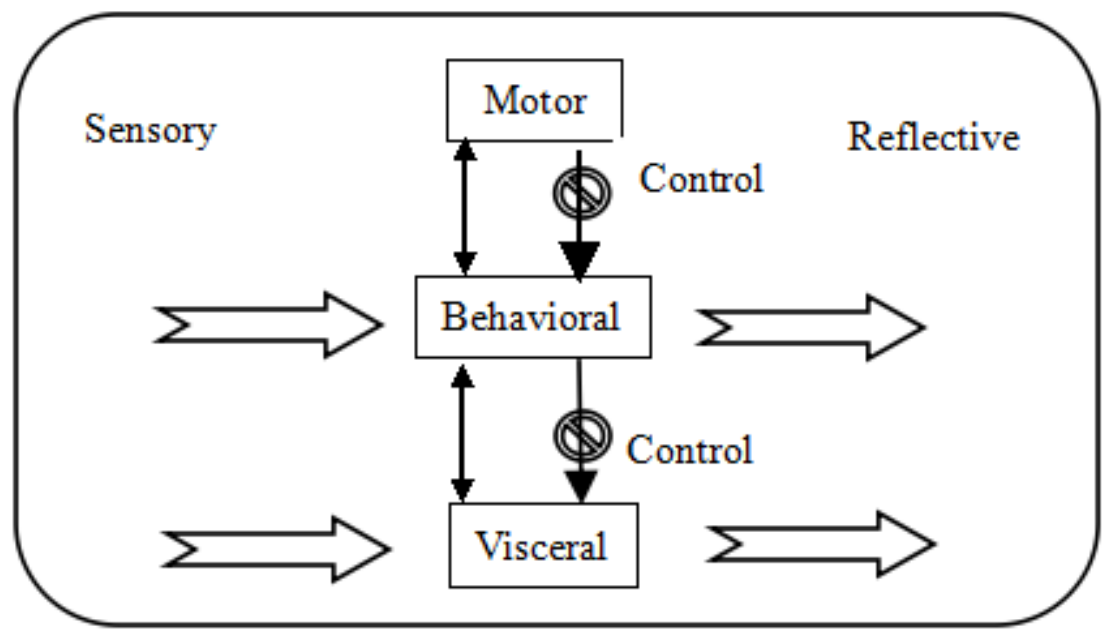

Fig. 2 Level of Emotional Design

Statistical model of hidden Markov model for Emotion Modeling

Due to the non-stationary random signal, the level of emotion and the emotion unobservable of sports students' sensibility signals, in the establishment of emotion model, will use the HMM process simulation of human psychological activity process, thus establishing the main emotion model HMM emotion.

Hidden Markov model H (hiddenMarkovModel, referred to as HMM) is a kind of statistical signal model,which was Markov signal source or Markov chain based on probability function, and proposed in twentieth Century at the end of the 60's and early 70's.It is a parametric representation, used for describe the probability model to describe the statistical properties of the random process. As shown in fig 3

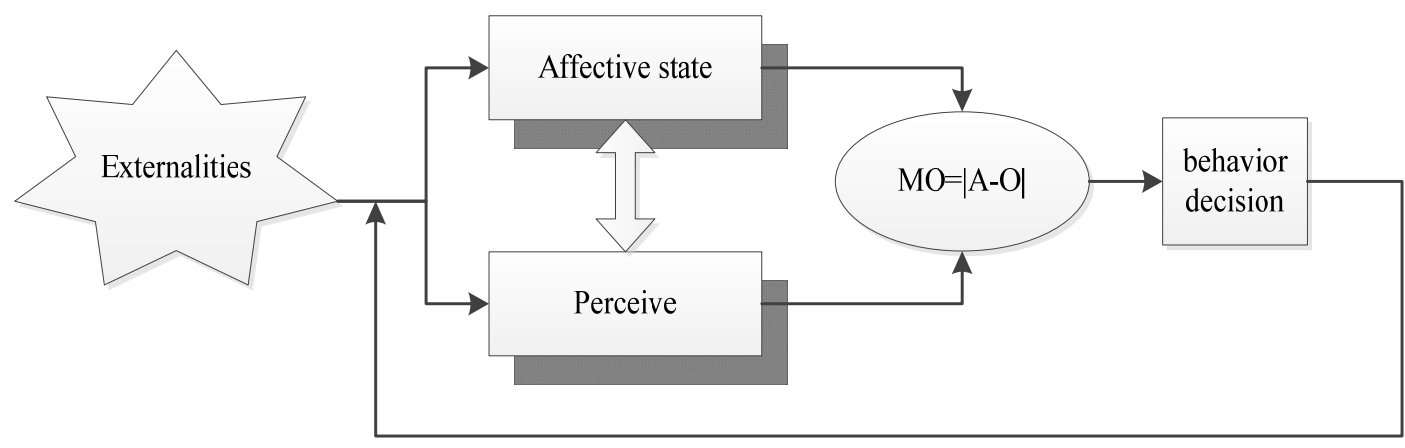

Fig 3 Hidden Markov model

The realization of emotional physical education model based on affective interaction

Modern distance physical education led to a profound change in education mode and education idea. It adopts advanced technology and means of education, will be through the computer network extends to every corner of the society, broke the learning time, space and personnel constraints, learners can learn independently at the appropriate time and place [3]. But based on the current PE teaching system in the concept of remote location, network 
system and the two-way interaction and other areas there are still shortcomings; especially in the two-way interaction, time and space of modern long-distance physical education separation makes learners cannot meet the emotional communication and the existence of emotion absence, this will affect the students' cognitive learning, even mental health, so and emotional compensation has important significance for guaranteeing the quality of teaching of modern long-distance physical education and improve the teaching effect of modern long-distance physical education. In this paper, with the emotional entropy as one of the constraints, to construct the initial state of emotion model of transfer matrix, provides a quantitative calculation method for virtual human character formation. And the analysis of affective model test results show that emotional reaction model simulation is consistent with the law of human affective behavior. Emotion model make stiff, cold machine into a "virtual human with", to the harmonious human-computer interaction a step forward [4].

In the design of the emotional model, first of all, we aimed at the most simple modeling:

(1)Hypothesis mood divided into: general, good mood, bad in three cases, the number of state corresponding to $\mathrm{M}=3$ in a hidden Markov model;

(2)Expression is divided into: expressionless, joy, no joy in three cases, corresponding the number of $\mathrm{N}=3$ in hidden Markov model to observe;

In the model, any state can be reached from any other state (a step), strictly speaking, properties of model is: any state can be reached from any other state in a finite number of steps. This model has the properties: each AIJ coefficient is positive, that is, for any $i, j$ are 错 误!未找到引用源。>0, only C), O (vIG), 0 (vIB) expressed respectively the emerging observe sequence value for the general, the good and the bad condition in the mood.

The probability of initial probability vector corresponding to the emotional model training before the start of each mood appears [5]. According to the history external stimulation of different and specific character, can be give the corresponding initial probability vector. The mood fluctuations decay with the increase of time, unless a new incentive. Thereforemost of the time people in a calm state of mind. When a virtual human at the first time and human interaction, because before this has not received any incentive, so at this point, it should be in a calm state of mind, with human-computer interaction, her emotions in accordance with the characteristics of human emotional response, and gradually accumulated and change. From the above analysis, we can determine when the virtual people without incentive effect on the initial probability

\section{Vector 错误!未找到引用源。 $=(\pi 1, \pi 2, \pi 3)=(1,0,0)$}

That calm state probability is 1 , the probability of state of happy and unhappy state are 0 . With the development of human-computer interaction, virtual human emotions in accordance with the characteristics of human emotional reaction, gradually accumulated, change. On the excitation signal of emotional awareness, we focus on stimulus. The consciousness form of the people although varied, but the human is like every other animal, accepted the most or the "reward" and "punishment" signal. "Reward" signal refers to the signal stimulation of the 
brain's pleasure center: "punish" refers to the signal stimulation of the brain's unhappy. Because of this, our emotional system mainly these two sensing signal response. In the course of programming, we will signal quantization into two dimensions, namely positive stimuli consisted of "a little reward" and "reward" signal, negative stimuli consisted of "a little punishment" and "punishment" signal. Accept different signal, corresponding to different emotion model parameters, as shown in Table 1

Table 1.different emotion model parameter

\begin{tabular}{|l|l|l|l|}
\hline Stimulus signals & $\begin{array}{l}\text { Emotional model } \\
\text { parameters }\end{array}$ & $\begin{array}{l}\text { Stimulus } \\
\text { signals }\end{array}$ & $\begin{array}{l}\text { Emotional model } \\
\text { parameters }\end{array}$ \\
\hline A little reward & $\mathrm{A}_{\mathrm{R}}(1), \mathrm{B}_{\mathrm{R}}(1), \mathrm{O}_{\mathrm{R}}(1)$ & A little punish & $\mathrm{A}_{\mathrm{P}}(1), \mathrm{B}_{\mathrm{P}}(1), \mathrm{O}_{\mathrm{P}}(1)$ \\
\hline Reward & $\mathrm{A}_{\mathrm{R}}(2), \mathrm{B}_{\mathrm{R}}(2), \mathrm{O}_{\mathrm{R}}(2)$ & Punish & $\mathrm{A}_{\mathrm{P}}(1), \mathrm{B}_{\mathrm{P}}(1), \mathrm{O}_{\mathrm{P}}(1)$ \\
\hline
\end{tabular}

We are in different combinations for emotional virtual human different stimulation, can get emotional reaction results of virtual human . Simulation study is performed using MATLAB software. We put the subliminal stimuli, divided into four types: "a little award", "award", "a little punishment" and "punishment". Thus, for each stimulus were corresponding a state transition probability matrix and visible symbol probability matrix. For the selection of initial value used for the model revaluation of the state transition rules matrix and visible symbol probability matrix, can be determined by experience, at the same time, want to consider the effect to external stimuli of various mentality emotion entropy, makes the role emotional entropy consistent with individual characteristics. We are located in accepting the "reward" signal, virtual human emotion mainly emotional state transition probability moment Array.

\section{CONCLUSION}

Motion recognition means distills and analyzes motion information of human, classifies and comprehends according to human's cognition and thinking manners, makes computer thinking and reasoning by using transcendent knowledge about human's emotion, then analyzes and comprehends human's emotion, it is a research field and development direction of affective computing.

\section{REFERENCE}

[1] Zuo,M.,Introduction to emotional interaction in network teaching. Audio-visual education in China,2,(2008).

[2 ]Hao, N., From the perspective of education psychology defects existing in the current network education. Electrochemical education research, 8,12,232-241(2002).

[3] Li,M.P., Affective computing theory and technology. Systems engineering and electronics. 7,(2003).

[4] Picard R W. Computers that Recognise and Respond to User Emotion: Theoretical and 
Practical Implications MIT Media Lab Tech Report, 1995.

[5] Abboud B .Davoine F.Dang M Statistical modeling for facial expression analysis and synthesis. IEEE International Conference on Image Processing. 2003 .pp:4--17. 